\title{
Telescope Array measurement of UHECR composition from stereoscopic fluorescence detection
}

\author{
Thomas Stroman $^{* a}$ and Yuichiro Tameda ${ }^{b}$ for the Telescope Array Collaboration ${ }^{\dagger}$ \\ ${ }^{a}$ University of Utah \\ E-mail: tstroman@physics.utah.edu \\ ${ }^{b}$ Kanagawa University \\ E-mail: tame@kanagawa-u.ac.jp
}

\begin{abstract}
The chemical composition of ultra-high-energy cosmic rays (UHECRs) affects the observable distribution of air-shower $X_{\max }$ values, the atmospheric slant depth at which the number of secondary shower particles reaches its maximum. The observed $X_{\max }$ distributions at various primary UHECR energies can be compared with the distributions predicted by detailed detector simulations for any assumed composition and high-energy hadronic interaction model. In this poster, we present measurements of $X_{\max }$ by the Telescope Array (TA) fluorescence detectors with stereoscopic shower reconstruction. We find that for all hadronic models considered, the TA 7-year fluorescence data set is consistent with a chiefly light UHECR composition.
\end{abstract}

The 34th International Cosmic Ray Conference,

30 July- 6 August, 2015

The Hague, The Netherlands

\footnotetext{
*Speaker.

${ }^{\dagger}$ For full author list and acknowledgments see http://www.telescopearray.org/images/papers/ICRC2015-authorlist.pdf
} 


\section{Introduction}

Ultra-high-energy cosmic rays (UHECRs; $E>10^{18} \mathrm{eV}$ ) are rare, so detailed study requires large indirect-detection experiments that use Earth's atmosphere as a calorimeter. The 700- $\mathrm{km}^{2}$ Telescope Array (TA) Experiment in west-central Utah, USA, is the largest UHECR detector in the northern hemisphere [1].

One of TA's primary science objectives is UHECR composition measurement. The extensive air shower produced by a "primary" UHECR incident on the atmosphere with a given energy reaches its maximum size at a slant depth $X_{\max }$ that systematically decreases with increasing primary mass. Composition measurement at TA consists of comparing the observed distribution of reconstructed $X_{\max }$ values with the distributions predicted by detailed Monte Carlo simulations that accurately model the detector aperture, under various assumptions of composition and the physics governing high-energy hadronic interactions; the latter must be extrapolated from laboratory measurements at much lower energies.

In this work, we present the observed $X_{\max }$ distribution from 7 years of operation, and compare it with simulated distributions using an identical analysis. We use CORSIKA to simulate the longitudinal development of protons and iron under several physics models (QGSJET-II-04 [2], QGSJET-II-03 [3], QGSJET-01c [4], EPOS LHC [5], and SIBYLL 2.1 [6]), and then simulate the detector response to a realistic flux in each model of either pure protons or pure iron. We perform $X_{\max }$ measurements using shower trajectories determined by stereoscopic triangulation, which is detailed in Section 2. We describe our simulations more completely in Section 3, followed by the presentation and discussion of our results in Sections 4 and 5, respectively.

\section{Stereo analysis}

The Telescope Array consists of three banks of fluorescence detector (FD) telescopes located 20-30 km apart on the periphery of a surface-detector array [7][8]. The FDs operate on clear, moonless nights and record the longitudinal development of air showers. When two FD sites record the same shower, the intersection of their respective shower-detector planes uniquely determines the position and orientation of the shower trajectory with high accuracy. In the case when all three FD sites observe a shower, an algorithm selects the best pair of sites based on the available plane-crossing angles.

The shower geometry reconstructed in this manner, combined with the current atmospheric density profile, determines the slant depth of the shower track observed by each FD pixel. We measure $X_{\max }$ via an inverse Monte-Carlo (IMC) technique, in which the parameters of a GaisserHillas ansatz are varied to find the shower profile that minimizes a $\chi^{2}$ comparison between observed and simulated photoelectrons either by pixel, in the case of the two FADC-based FD stations, or by laterally integrated angular bin, for profiles observed by the third FD station, built from refurbished sample-and-hold equipment originally used in the High Resolution Fly's Eye.

Energy reconstruction begins with integration of the best-fit Gaisser-Hillas profile, weighted by a self-consistent energy-deposit model, to obtain the calorimetric energy. To arrive at the initial energy, compensation for "missing" energy (production of muons and neutrinos) uses a correction calculated from analysis of QGSJET-II-03 protons. 


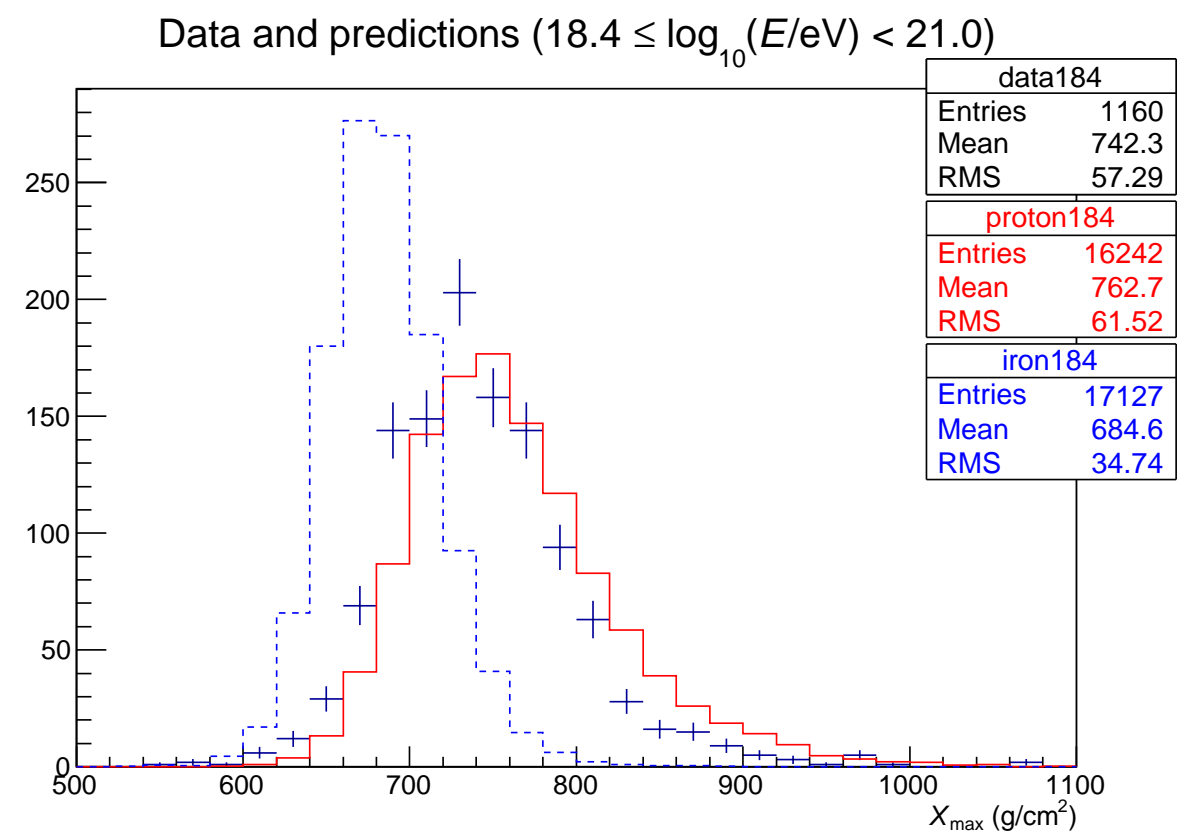

Figure 1: Comparison of the reconstructed $X_{\max }$ distribution from stereo analysis of 7 years' data (black points) with the QGSJET-II-03 predictions for a composition that is either 100\% proton (red solid line) or $100 \%$ iron (blue dashed line). The minimum energy is $10^{18.4} \mathrm{eV}$.
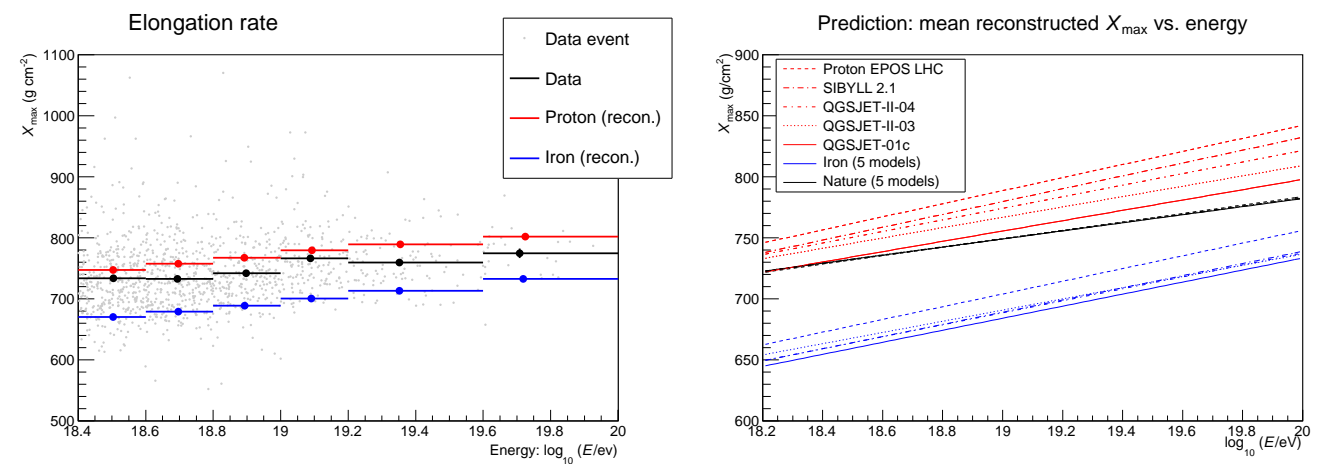

Figure 2: a. The evolution of the mean reconstructed $X_{\max }$ with reconstructed primary energy, of 7 years' stereo data (black points) compared with QGSJET-II-03 predictions for protons (red points) and iron (blue points). b. Linear fits to the mean- $X_{\max }$ observation (black line) and predictions for protons (red lines) and iron (blue lines) from all five hadronic-interaction models considered. The near-indistinguishability of the five black lines demonstrates the insensitivity of the data reconstruction to the choice of model.

Each FD site that observes a shower attempts to reconstruct its profile independently, which results in up to three successful measurements of Gaisser-Hillas parameters for the same shower. When two or three measurements pass all monocular-profile quality cuts, we use the unweighted average values of $X_{\max }$ and $\log _{10}(E / \mathrm{eV})$. If only one measurement survives the cuts, it is only admitted to the final data set if it passes an additional quality cut based on pattern-recognition analysis, which was developed for FD+surface detector "hybrid" profile reconstruction and is described in detail elsewhere [9]. 


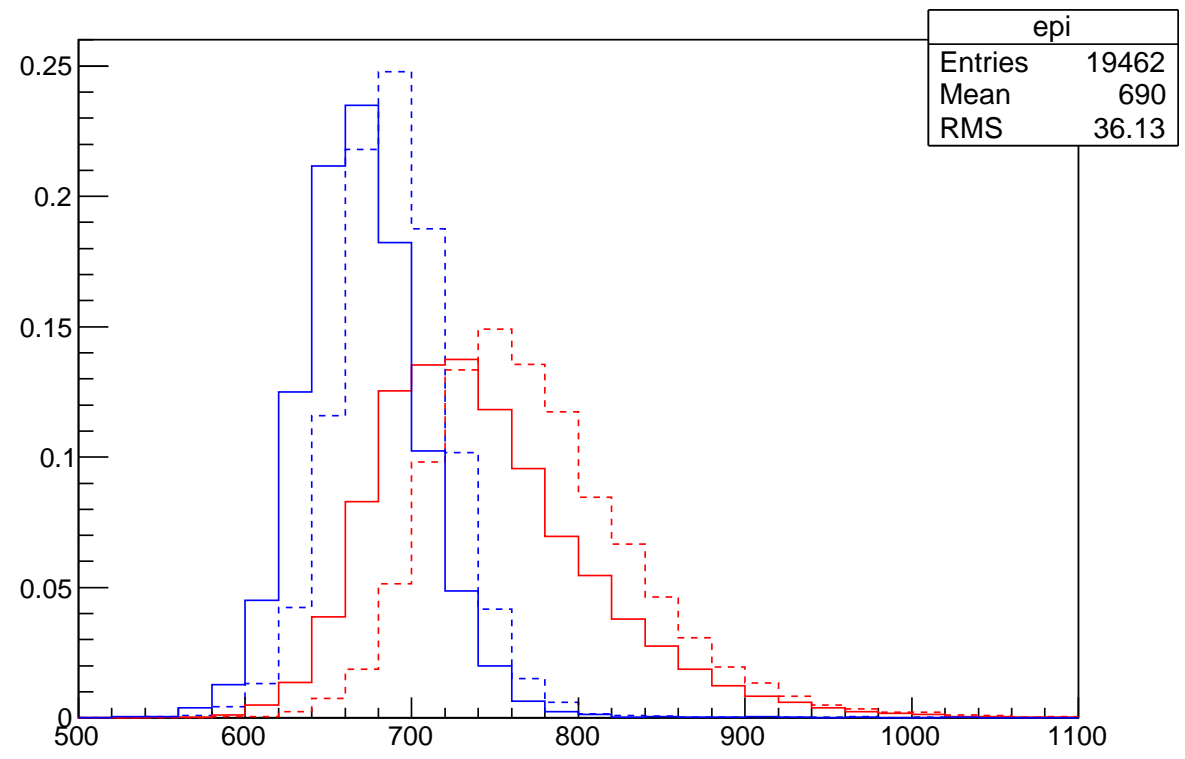

Figure 3: The proton (red) and iron (blue) predicted $X_{\max }$ distributions for primary energies in the range $10^{18.6} \leq E / \mathrm{eV}<10^{18.8}$, according to QGSJET-01c and EPOS LHC, respectively the shallowest and deepest of all hadronic-interaction models considered. The models are not in disagreement about the shape of a given particle species' distribution; it is only the overall slant depth that differs between even the most disparate predictions.

\section{Simulation procedure}

The prediction of the $X_{\max }$ distribution for each composition and model uses a library of Gaisser-Hillas fits to the longitudinal output of CORSIKA shower simulations at several values of primary energy and inclination. Detector simulation is performed for all nights when at least two FDs were operating from 2007-11-04 through 2014-11-01, with shower trajectories selected at random according to an isotropic distribution with zenith angles $\theta \leq 80^{\circ}$. Shower energies are chosen starting from $10^{17.7} \mathrm{eV}$ and following the published HiRes spectrum [10]. Detector response is then simulated, including fluorescence and Cherenkov light production, radiation transport, optical acceptance, and detector electronics simulation including night-sky background noise.

The simulation output includes artificial raw data, suitable for the entire processing and analysis chain applied to natural night-sky data. This chain includes identification of shower-detector planes, inter-FD coincidence detection, stereo geometry calculation, and profile reconstruction.

\section{Results}

We show our $X_{\max }$ distributions for $E \geq 10^{18.4} \mathrm{eV}$ and the QGSJET-II-03 predictions for protons and iron in Figure 1. Figure 2 shows the mean of the observed and simulated distributions in several energy bins, and linear fits to these values from all physics models to illustrate the relationships among the various predictions. Finally, Figure 3 shows the consistent shape of the distributions among different models even when the predicted means differ widely. 


\section{Discussion and conclusions}

Although iron's cosmic abundance, nuclear stability, and large electric charge make it an attractive candidate for acceleration to ultra-high energies from a theoretical standpoint, the results shown in Section 4 demonstrate that the $X_{\max }$ distribution observed by stereo analysis of TA data does not support iron at any energy above $10^{18.4} \mathrm{eV}$, regardless of which model of hadronic physics is assumed. The pure-proton composition is especially attractive when combined with QGSJET-01c, but the agreement is less clear when post-LHC models are considered. This applies marginally to QGSJET-II-04, and much more strongly to EPOS LHC, and also to the pre-LHC model SIBYLL 2.1; LHC corrections to SIBYLL are expected to further widen the difference from TA data [11]. The latter models' protons fail to predict our observations under even the most generous invocation of systematic uncertainty.

On average, our reconstruction of $X_{\max }$ and energy are respectively accurate to better than $25 \mathrm{~g} / \mathrm{cm}^{2}$ and $7 \%$. The systematic uncertainty on these TA $X_{\max }$ measurements is approximately $15 \mathrm{~g} / \mathrm{cm}^{2}$. Much of this originates in the atmospheric models used, both for the density profile and the aerosol distribution.

Further work on this analysis, currently in progress, will help to clarify the extent to which the UHECR composition is explained by protons alone. A contribution from other chemical constituents may help to bring various moments of the observed and simulated $X_{\max }$ distributions into agreement, but we are also exploring the use of statistically robust comparisons that consider the entire distribution. In particular, the Cramér-von Mises test statistic, combined with the value of whatever artifically imposed offset minimizes it, shows great promise for quantifying the role played by intermediate-mass elements in the UHECR flux.

\section{References}

[1] J. Matthews et al., Overview of the Telescope Array Experiment, in Proceedings of the 31st ICRC (Łódź), p. icrc1386, 2009.

[2] S. Ostapchenko, QGSJET-II: physics, recent improvements, and results for air showers, in European Physical Journal Web of Conferences, vol. 52 of European Physical Journal Web of Conferences, p. 2001, June, 2013.

[3] S. Ostapchenko, QGSJET-II: towards reliable description of very high energy hadronic interactions, Nuclear Physics B Proceedings Supplements 151 (Jan., 2006) 143-146, [hep-ph/ 0412332 ].

[4] N. N. Kalmykov, S. S. Ostapchenko, and A. I. Pavlov, Quark-Gluon-String Model and EAS Simulation Problems at Ultra-High Energies, Nuclear Physics B Proceedings Supplements 52 (Feb., 1997) 17-28.

[5] T. Pierog, I. Karpenko, J. M. Katzy, E. Yatsenko, and K. Werner, EPOS LHC : test of collective hadronization with LHC data, ArXiv e-prints (June, 2013) [arXiv : 1306.0121 ].

[6] E.-J. Ahn, R. Engel, T. K. Gaisser, P. Lipari, and T. Stanev, Cosmic ray interaction event generator SIBYLL 2.1, Phys. Rev. D 80 (Nov., 2009) 094003, [arXiv: 0906.4113 ].

[7] H. Tokuno et al., New air fluorescence detectors employed in the Telescope Array experiment, Nuclear Instruments and Methods in Physics Research A 676 (June, 2012) 54-65, [arXiv: 1201.0002 ]. 
[8] T. Abu-Zayyad et al., The energy spectrum of Telescope Array's Middle Drum detector and the direct comparison to the High Resolution Fly's Eye experiment, Astroparticle Physics 39 (Dec., 2012) 109-119, [arXiv:1202.5141].

[9] R. U. Abbasi et al., Study of Ultra-High Energy Cosmic Ray composition using Telescope Array's Middle Drum detector and surface array in hybrid mode, Astroparticle Physics 64 (Apr., 2015) 49-62, [arXiv: 1408.1726$]$.

[10] R. U. Abbasi et al., First Observation of the Greisen-Zatsepin-Kuzmin Suppression, Physical Review Letters 100 (Mar., 2008) 101101, [astro-ph/0703099].

[11] E. Ahn, R. Engel, T. K. Gaisser, P. Lipari, F. Riehn, and T. Stanev, LHC Update of the Hadronic Interaction Model SIBYLL 2.1, in International Cosmic Ray Conference, 33rd ICRC (Rio de Janeiro) abstract 0803, 2013. 